\title{
Evolution of dust properties in an interstellar filament
}

\author{
B. Stepnik ${ }^{1,2}$, A. Abergel ${ }^{1}$, J.-P. Bernard ${ }^{1}$, F. Boulanger ${ }^{1}$, L. Cambrésy ${ }^{3}$, M. Giard ${ }^{2}$, A. P. Jones ${ }^{1}$, G. Lagache ${ }^{1}$, \\ J.-M. Lamarre ${ }^{1}$, C. Meny ${ }^{2}$, F. Pajot ${ }^{1}$, F. Le Peintre ${ }^{1}$, I. Ristorcelli ${ }^{2}$, G. Serra ${ }^{2}$, and J.-P. Torre ${ }^{4}$ \\ ${ }^{1}$ Institut d'Astrophysique Spatiale (IAS), Bât. 121, Université Paris XI, 91405 Orsay Cedex, France \\ 2 Centre d'Étude Spatiale des Rayonnements (CESR), 9 Av. Colonel Roche, BP 4346, 31028 Toulouse, France \\ 3 Observatoire Astronomique de Strasbourg, UMR 7550, Université Louis Pasteur, 67000 Strasbourg, France \\ ${ }^{4}$ Service d'Aéronomie du CNRS (SA), BP 3, 91371 Verrières Le Buisson, France
}

Received 16 January 2001 / Accepted 8 March 2002

\begin{abstract}
We present submillimetre observations obtained using the balloon-borne experiment PRONAOS/SPM, from 200 to $600 \mu \mathrm{m}$ with an angular resolution of $2-3.5^{\prime}$, of a quiescent dense filament (typically $A_{V} \sim 4$ ) in the Taurus molecular complex. This filament, like many other molecular clouds, presents a deficit in its IRAS $I_{60 \mu \mathrm{m}} / I_{100 \mu \mathrm{m}}$ flux ratio in comparison with the diffuse interstellar medium.

We show, from the combination of the PRONAOS/SPM and IRAS data, that, inside the filament, there is no evidence for emission from the transiently heated small particles responsible for the $60 \mu \mathrm{m}$ emission, and that the temperature of large grains in thermal equilibrium with the radiation field is reduced in the inner parts of the filament. The temperature is as low as $12.1_{-0.1}^{+0.2} \mathrm{~K}$ with $\beta=1.9 \pm 0.2$ (or $12.0_{-0.1}^{+0.2} \mathrm{~K}$ using $\beta=2$ ) toward the filament centre. These phenomena are responsible for the IRAS colour ratio observed toward the filament.

In order to explain this cold temperature, we have developed a model for the emission from the filament using star counts from the 2MASS catalog as an independent tracer of the total column density and a radiative transfer code. We first use the optical properties of the dust from the standard model of Désert et al. (1990). The computed brightness profiles fail to reproduce the data inside the filament, showing that the dust properties change inside the filament. An agreement between data and model can be found by removing all the transiently heated particles from the densest parts of the filament, and multiplying the submillimetre emissivity by a significant factor, $3.4_{-0.7}^{+0.3}$ (for typically $n_{H}>3 \pm 1 \times 10^{3} \mathrm{~cm}^{-3}, A_{V}>2.1 \pm 0.5$ ).

We show that grain-grain coagulation into fluffy aggregates may occur inside the filament, explaining both the deficit of small grain abundance and the submillimetre emissivity enhancement of the large grains.
\end{abstract}

Key words. ISM: clouds - ISM: dust, extinction - infrared: ISM - radiative transfer - radio continuum: ISM

\section{Introduction}

The interstellar dust is made of several components differing in their chemical composition, structure and size (e.g. Mathis 1990). The smallest interstellar dust particles (large aromatic molecules and very small grains, sizes $\lesssim 15 \mathrm{~nm}$ ) essentially emitting at wavelengths below $100 \mu \mathrm{m}$, are transiently heated in the interstellar radiation field (ISRF) (Sellgren et al. 1985). The largest interstellar dust grains are in thermal equilibrium with the radiation field. These grains emit predominantly at wavelengths larger than $100 \mu \mathrm{m}$ and peak around $150 \mu \mathrm{m}$ in the diffuse medium. The observed emissivity (Boulanger et al. 1996) is similar to the value predicted by the grain model of Draine \& Lee (1984) which constituted of a mixture of compact graphite

Send offprint requests to: A. Abergel,

e-mail: alain. abergel@ias.fr and silicate grains. However, this simple description does not take into account the spatial variations of the dust properties in our galaxy revealed by IRAS and COBE.

IRAS data show that the colour of the dust emission in the ISM varies from place to place within the same molecular complex (Boulanger et al. 1990). In particular, the colour ratio $I_{60 \mu \mathrm{m}} / I_{100 \mu \mathrm{m}}$ can decrease dramatically from diffuse to molecular regions (Laureijs et al. 1991; Abergel et al. 1994, 1996). COBE data show that the dust temperature is lower $(14.5 \mathrm{~K})$ in dense molecular clouds with low mass star-forming activity than in diffuse regions (Lagache et al. 1998). Unfortunately, the physical properties of the cold dust are not well characterised because of the limitations of both the IRAS and COBE instrumentation. IRAS was limited to wavelengths shorter than $100 \mu \mathrm{m}$ while COBE had a poor angular resolution. 
The balloon borne experiment PRONAOS/SPM (Lamarre et al. 1994) combined a spatial resolution of $2-3.5^{\prime}$ with an extended spectral coverage from 200 to $600 \mu \mathrm{m}$. Data from PRONAOS/SPM combined with data from IRAS enable us to better understand the nature of the cold dust in the ISM. Recently, observations with PRONAOS/SPM have constrained the dust properties in cold condensations in the Orion complex (Ristorcelli et al. 1998) and in a cirrus cloud in the Polaris flare (Bernard et al. 1999).

In this paper, we analyse an individual cloud in the Taurus complex. IRAS data of this cloud present large spatial variations of the colour ratio $I_{60 \mu \mathrm{m}} / I_{100 \mu \mathrm{m}}$. Several hypotheses have been suggested to account for these variations (Laureijs et al. 1991; Abergel et al. 1994), e.g., a temperature decrease due to the extinction of the incident radiation field, variations in emissivity of grains in thermal equilibrium with the radiation field, and variations in the abundance and/or the optical properties of Very Small Grains (VSGs). In order to distinguish between these various hypotheses and to understand the evolution of dust properties from diffuse to dense ISM, we combine dust modelling with radiative transfer calculations to explain the PRONAOS/SPM and IRAS observations.

The paper is organised as follows. In Sect. 2, we present the submillimetre observations obtained using the PRONAOS/SPM instrument. In Sect. 3, we describe the data processing. In Sect. 4 , we analyse the submilimetre and $A_{V}$ profiles of the cloud. In Sect. 5, we compare the observed emission profiles with an emission model for the cloud. We show that the presence of standard grains inside the filament is not compatible with our data and we propose non-standard dust optical properties in order to reproduce our observations. In Sect. 6, we discuss the presence and the optical properties of non-standard grains inside the filament. Finally, in Sect. 7, we conclude and summarise our results.

\section{Submillimetre observations}

\subsection{Selection of the filament}

The Taurus molecular cloud complex, at a distance of $140 \mathrm{pc}$, is one of the nearest low mass star forming regions (Elias 1978; Kenyon et al. 1994). The IRAS images shows a network of filamentary structures, with typical widths of $\sim 1 \mathrm{pc}$, which include almost all star forming regions (Abergel et al. 1994). The extended emission has an uniform IRAS colour ratio $I_{60 \mu \mathrm{m}} / I_{100 \mu \mathrm{m}}=0.15$, while the filamentary structures present a deficit of $70-100 \%$ of this ratio (Abergel et al. 1994). The residual emission $I_{\text {excess }}^{100}=I_{v}(100)-I_{v}(60) / 0.15$ is the IR emission excess, and emphasises the filamentary structures. It is spatially correlated with the velocity integrated ${ }^{13} \mathrm{CO}(J=1-0)$ molecular maps, indicating that the deficit of emission at $60 \mu \mathrm{m}$ comes from dense structures $\left(n_{H}>10^{3} \mathrm{~cm}^{-3}\right)$. Using largescale DIRBE data, Lagache et al. (1998) have shown that the dense material of the Taurus complex is colder $(\sim 13 \mathrm{~K})$ than the diffuse medium surrounding it $(\sim 17.5 \mathrm{~K})$. This paper presents observations of a dense filament in Taurus, centred at the position: $\alpha_{2000}=4^{\mathrm{h}} 18^{\mathrm{m}} 50^{\mathrm{s}}, \delta_{2000}=25^{\circ} 19^{\prime} 15^{\prime \prime}$ (Fig. 1). In the IRAS data this filament shows a strong deficit of emission at $60 \mu \mathrm{m}$ compared to the emission at $100 \mu \mathrm{m}$.

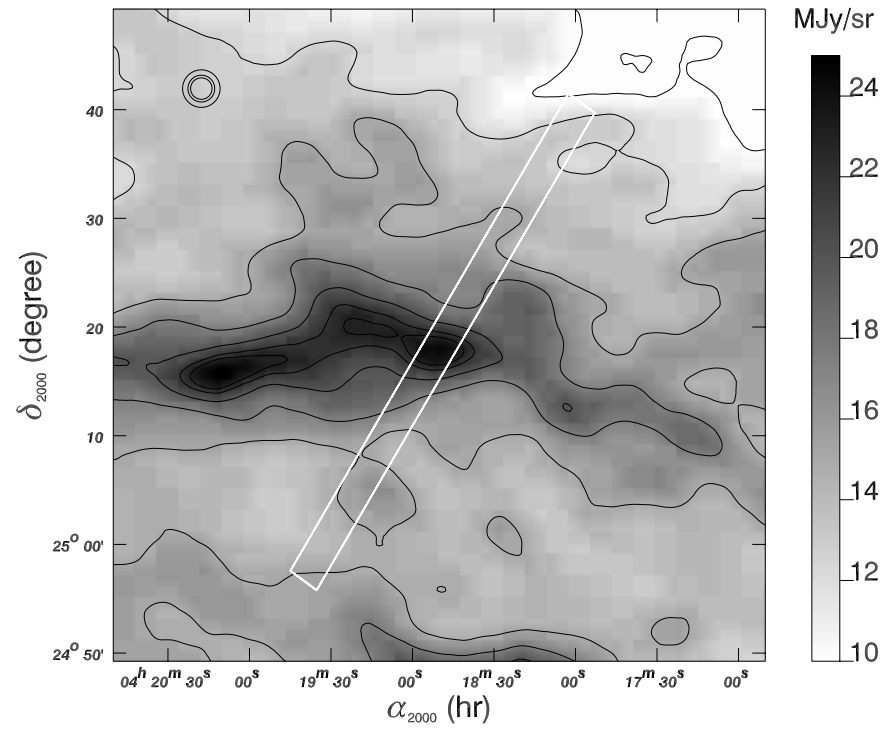

Fig. 1. $100 \mu$ m IRAS map of the Taurus filament. The white box shows the region covered by the optical axis of PRONAOS/SPM (not including the instrument beam size). The PRONAOS/SPM beam sizes at each wavelength are shown by the circles in the top left corner of the figure. The $100 \mu \mathrm{m}$ IRAS contours are given from 11 to $23 \mathrm{MJy} / \mathrm{sr}$ with a step of $2 \mathrm{MJy} / \mathrm{sr}$.

Table 1. Characteristics of the PRONAOS/SPM and IRAS spectral bands for our observation. The IRAS sensitivity is the rms noise of the ISSA map.

\begin{tabular}{ccccc}
\hline \hline Instrument & $\begin{array}{c}\lambda \\
(\mu \mathrm{m})\end{array}$ & $\begin{array}{c}\Delta \lambda \\
(\mu \mathrm{m})\end{array}$ & $\begin{array}{c}F W H M \\
\left({ }^{\prime}\right)\end{array}$ & sensitivity \\
\hline IRAS & 12 & 6.5 & 3.4 & $0.06 \mathrm{MJy} / \mathrm{sr}$ \\
(ISSA) & 25 & 11 & 3.8 & $0.1 \mathrm{MJy} / \mathrm{sr}$ \\
& 60 & 40 & 3.5 & $0.1 \mathrm{MJy} / \mathrm{sr}$ \\
PRONAOS & 200 & 37 & 4.2 & $0.5 \mathrm{MJy} / \mathrm{sr}$ \\
(SPM) & 260 & 100 & 2.0 & $30 \mathrm{MJy} / \mathrm{sr} / \mathrm{Hz}^{\frac{1}{2}}$ \\
& 360 & 200 & 2.5 & $7 \mathrm{MJy} / \mathrm{sr} / \mathrm{Hz}^{\frac{1}{2}}$ \\
& 580 & 660 & 3.5 & $3 \mathrm{MJy} / \mathrm{sr} / \mathrm{Hz}^{\frac{1}{2}}$ \\
\hline
\end{tabular}

\subsection{PRONAOS/SPM instrument}

The observations were performed with the PRONAOS experiment $^{1}$ (PROgramme National d'AstrOnomie Submillimétrique). PRONAOS was a stratospheric balloon-borne two meter telescope devoted to submillimetre astronomy. The balloon flew three times in 1994, 1996 and 1999. PRONAOS could be pointed to a relative accuracy of $5^{\prime \prime}$ (Buisson \& Durand 1990; Serra et al. 1997). The focal plane instrument SPM (Spectro Photomètre Multibande, see Lamarre et al. 1994) was a single beam multi-band photometer. It performed simultaneously measurements in four bands spanning a wavelength range from 200 to $580 \mu \mathrm{m}$ (Table 1). The detector system used bolometers cooled to $0.3 \mathrm{~K}$ using closed cycle ${ }^{3} \mathrm{He}$

1 This experience was conducted by CNES, and designed and built in a large national cooperation between CNES and various CNRS laboratories. 
coolers (Torre \& Chanin 1985) inside a liquid ${ }^{4} \mathrm{He}$ cryostat. This system provided an intrinsic noise-equivalent power of about $10^{-15} \mathrm{~W} \mathrm{~Hz}^{-1 / 2}$, close to the photon noise. The atmospheric residual emission was reduced using an oscillating mirror, which allows beam switching on the sky. The measured signal was the difference between the sky flux at $+3^{\prime}$ and $-3^{\prime}$, at constant elevation, and along the scan direction. For this reason, only emission gradients were detected. Reconstruction of the astrophysical signal requires deconvolution from the dual beam pattern, as is the case for ground based observations in the submillimetre continuum (Sect. 2.4).

The photometric calibration was provided by ground measurements, two in flight black bodies, and planet observations. The final accuracy of this calibration was better than $8 \%$ absolute, and better than 5\% from channel to channel (Pajot et al. 2002).

\subsection{Observing strategy}

The Taurus cloud observations were carried out during the second PRONAOS/SPM flight, September 23rd 1996, from Fort Sumner, New-Mexico (USA), as part of a NSBF-NASA launch campaign. PRONAOS/SPM was a single beam instrument, therefore in order to obtain a map we have to scan the source with an "on the fly" mode. 21 scans were performed at a constant elevation, with a length of $50^{\prime}$, and a distance between each scan of $2^{\prime \prime}$. Gondola oscillations and sky rotation during scanning results in a map of $3^{\prime} \times 50^{\prime}$ of the target (Fig. 1). Note that the width of the map is slightly larger than the beam size. Despite the gondola oscillations, which enlarge the map size, the final position reconstruction, which is based upon stellar sensor information (including rotation), has a relative accuracy of $\sim 5^{\prime \prime}$ (Buisson \& Durand 1990). The scan speed was constant and equal to $36^{\prime \prime} / \mathrm{s}$. The total duration of the observation was $40 \mathrm{~min}$.

\section{Data processing}

\subsection{PRONAOS/SPM data}

The data processing has been performed in six independent steps, for each wavelength: (1) deglitching, (2) data averaging and filtering, (3) subtraction of instrumental drifts, (4) deconvolution, (5) calibration and (6) construction of equatorial maps.

Step 1: we have separated the signal into blocks of 50 data points. We have computed the average and the root mean square $(\sigma)$ of each block after discarding the $30 \%$ greater and the $30 \%$ lower data points. Points above 10 sigma have been considered as glitches and have been discarded.

Step 2: in order to reduce the high frequency noise due to the electronic readout, we have convolved the signal with a Hamming function computed on twenty data points (corresponding to $\sim 40^{\prime \prime}$ ).

Step 3: long-term fluctuations due to atmospheric effects and instrumental drifts have to be removed. The beam switching scanning strategy implies that the average of a scan must be near zero if pointing errors and gradients on the sky at the edges of the map are low, as in our case. We have computed the median signal for every scan, and subtract a cubic spline function fitted to these values to the signal.

Step 4: we have deconvolved each scan using a Fourier deconvolution method (Emerson et al. 1979). Due to the deconvolution, the absolute emission of the sky is filtered out, and the edges of each scan are arbitrarily set to zero. The method removes signal information near the modulation frequency. As a result, artificial oscillations may appear on the deconvolved signal due to aliasing. Lower signal-to-noise ratios induce higher oscillations. At 60 and $580 \mu \mathrm{m}$ the oscillations are strongest because the uncertainties are dominant.

Step 5: we have calibrated our data with two in flight black bodies. A calibration measurement was performed just before and after our observations. We have averaged these two observations to derive the applied calibration parameters.

Step 6: finally, we have computed the coordinates of each data point, using the pointing information from the stellar sensor and the information on the balloon oscillations. The restored pointing accuracy is about 5" (Buisson \& Durand 1990). We have projected the data points onto the coordinate grid of the IRAS map. Typically, we have averaged $\sim 60$ measurement points in one $30^{\prime \prime}$ pixel. The size of the final maps is $3^{\prime} \times 50^{\prime}$.

\subsection{IRAS and COBE data}

To complement our data we have used IRAS data (ISSA maps, Wheelock et al. 1998) and COBE/DIRBE data (ZSMA maps, Hauser et al. 1997) of the cloud.

PRONAOS/SPM performs differential measurements, thereby filtering out structures larger than the scan length of $50^{\prime}$. In order to account for similar filtering in the IRAS observations, we simulated PRONAOS/SPM observations on the IRAS maps. For each measurement point, we modulated the IRAS map in the same direction, at the same coordinate position and with the same modulation distance as for the PRONAOS/SPM observations. Then we deconvolved the IRAS signal and generated maps. The oscillations in the $60 \mu \mathrm{m}$ IRAS profiles are induced (see below) by residual stripes on the IRAS maps.

\subsection{Submillimetre profiles}

We have generated $3^{\prime} \times 50^{\prime}$ brightness maps of the cloud at 12 , $25,60,100,200,260,360$ and $580 \mu \mathrm{m}$. In order to maximise the signal-to-noise ratio, we have computed profiles across the cloud by averaging the maps in a direction parallel to the long axis of the cloud (Fig. 2). The IRAS brightness uncertainties have been computed from the quadratic sum of the IRAS calibration errors ( $10 \%$ of the signal) and the rms in a $20^{\prime} \times 20^{\prime}$ empty field centred at $\alpha_{2000}=4^{\mathrm{h}} 14^{\mathrm{m}} 28^{\mathrm{s}}, \delta_{2000}=26^{\circ} 08^{\prime} 02^{\prime \prime}$ (Table 1). Figure 2 shows that no significant emission is detected at 12 and $25 \mu \mathrm{m}$. Towards the filament centre, the signal is less than 1 and $2 \sigma$ at 12 and $25 \mu \mathrm{m}$ respectively. Therefore, we will not include the 12 and $25 \mu \mathrm{m}$ data in our analysis.

We see in Fig. 2 that the cloud is composed of a bright filament which appears on top of a large-scale surrounding envelope. The filament is visible at all wavelengths except at $60 \mu \mathrm{m}$. 


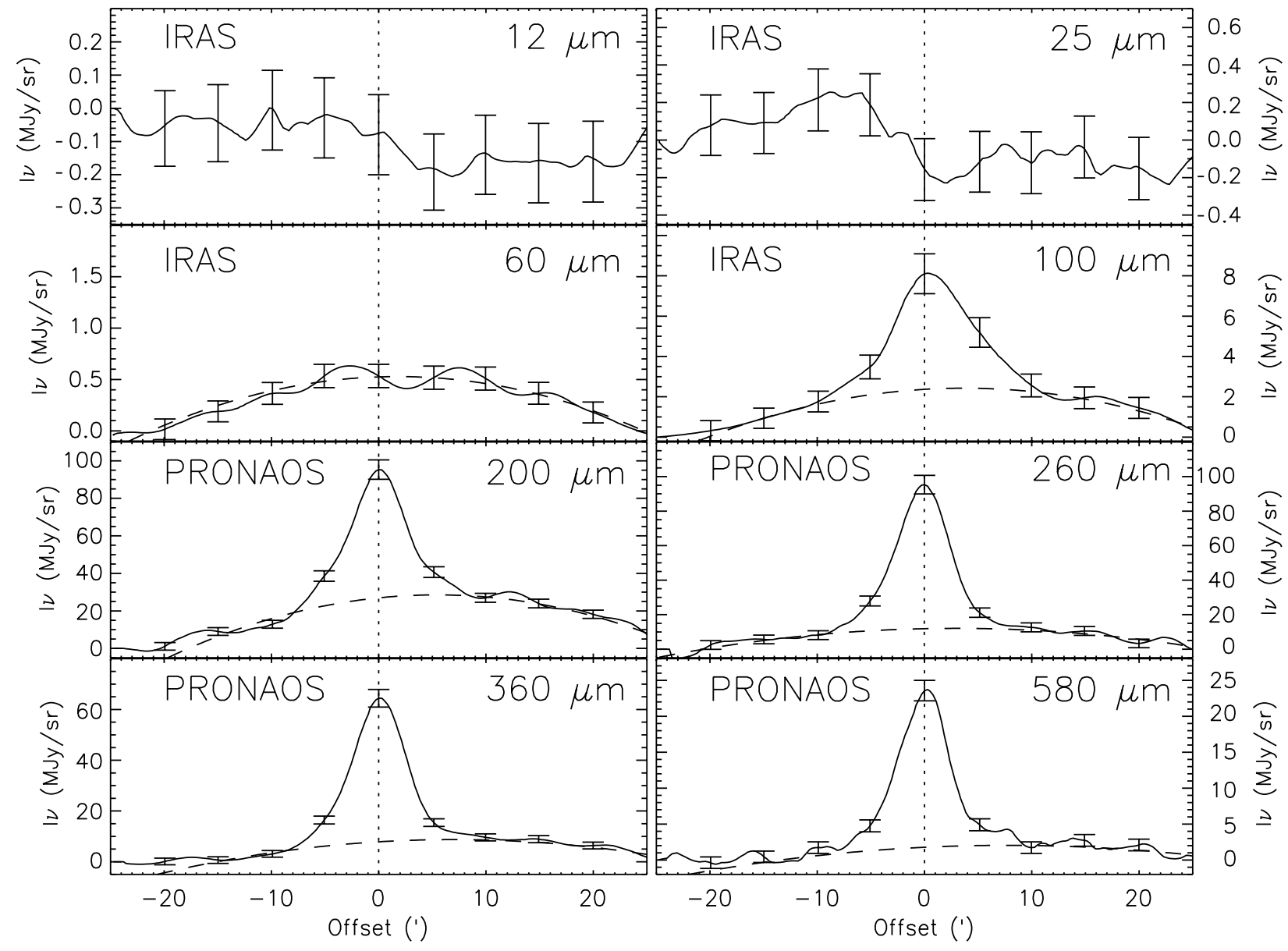

Fig. 2. Average brightness profiles of the cloud with \pm 1 sigma error bars. The solid lines are the observed profiles at the angular resolution of Table 1. The cloud emission is decomposed into the central filament and the surrounding envelope (fitted by a second order polynomial, dashed lines). The cloud is scanned from negative to positive offsets, and with a decreasing declination (see Fig. 1).

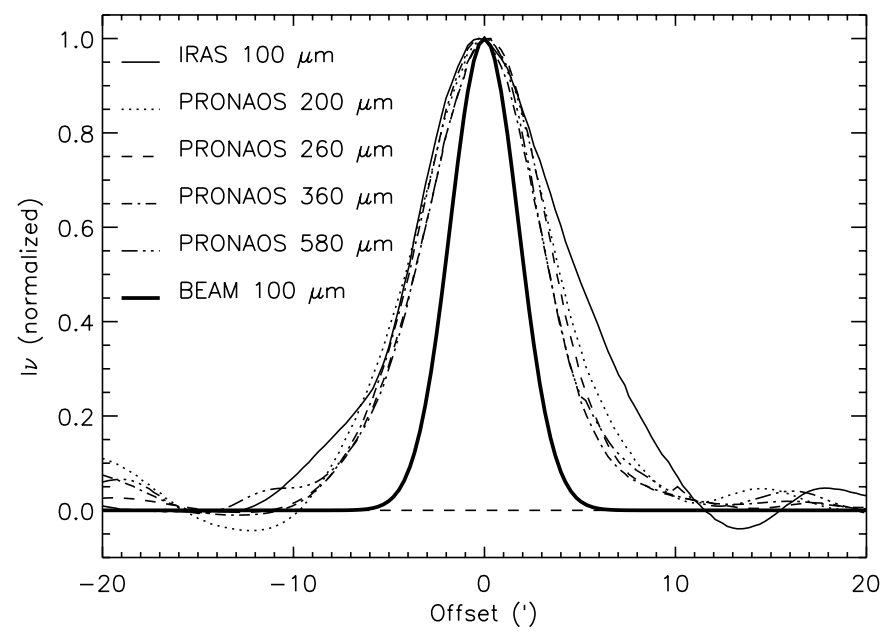

Fig. 3. Brightness profiles of the filament after subtraction of the surrounding envelope. The surface brightness is normalised to the maximum of each band and the profile resolution is degraded to $4.2^{\prime}$, which is the $F W H M$ of the IRAS $100 \mu \mathrm{m}$ beam.

Away from the bright filament, there is no evidence for smallscale structure in the profiles, except for the instrumental oscillations (Sect. 3.1).
To compare the brightness profiles, we must have the same resolution in each band. The IRAS beam sizes have been determined on a point source near the filament $\left(2.5^{\circ}\right.$ away from the filament centre, $\alpha_{2000}=04^{\mathrm{h}} 29^{\mathrm{m}} 22^{\mathrm{s}}, \delta_{2000}=24^{\circ} 33^{\prime} 27^{\prime \prime}$ ). The PRONAOS/SPM beam sizes have been measured in flight on Saturn (Pajot et al. 2002). Table 1 presents the angular resolution of each band in a direction perpendicular to the long axis of the cloud, which is the direction of the profiles presented in Fig. 2. We have degraded the resolution of all the data to that of the $100 \mu \mathrm{m}$ band $\left(4.2^{\prime}\right)$ by convolving the data with Gaussian functions. Applying a $\chi^{2}$ minimisation method we obtained Gaussian functions of $2.36^{\prime}, 3.65^{\prime}, 3.65^{\prime}, 3.51^{\prime}$ and $2.85^{\prime} \mathrm{FWHM}$ to transform the profiles at 60, 200, 260, 360 and $580 \mu \mathrm{m}$ into that at $100 \mu \mathrm{m}$, respectively, with an accuracy better than $1 \%$.

\subsection{Extinction data from star counts}

We have used the 2MASS $J$ band star catalog (Cutri et al. 2000), and applied the star count method developed by Cambrésy (1999) to derive an $A_{V}$ map. We make two assumptions:

- All stars are located behind the cloud. This is justified because the cloud is very close (140 pc, see Elias 1978; 


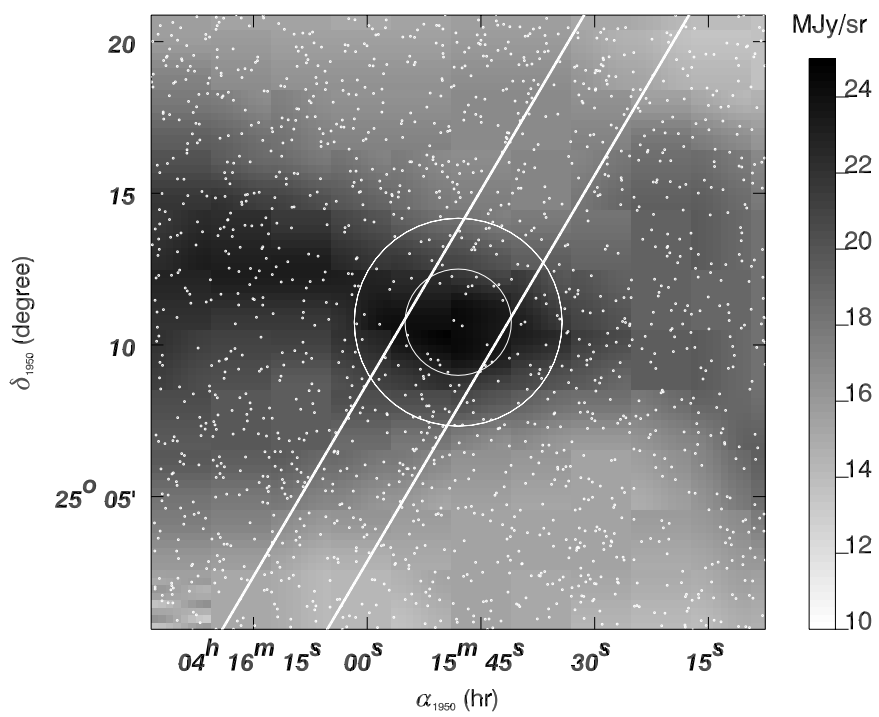

Fig. 4. IRAS map at $100 \mu \mathrm{m}$ around the filament centre with the position of the 2MASS stars used in the star count method. The white lines show the region observed by PRONAOS/SPM. The circles have $3.5^{\prime}$ and $7^{\prime}$ diameters.

Kenyon et al. 1994), and the comparison of the $J$ and $K$ magnitudes for the stars shows that most of the stars are redder near the filament centre than in the outer parts.

- The stars have an uniform apparent distribution in the abscence of extinction. This assumption is justified when looking at the stellar distribution outside the filament (Fig. 4).

Following Cambrésy (1999) we use an adaptive grid with a fixed number of 20 stars in each cell. Then a filtering method involving a wavelet decomposition filters the noise without resampling the data. The counts are corrected for the variation of the background stellar density with galactic latitude, assuming an exponential law for the stellar density. Finally, $A_{J}$ values are converted into $A_{V}$ values using $A_{J} / A_{V}=0.282$, from Cardelli et al. (1989). The resulting $A_{V}$ map has an angular resolution of $3.5^{\prime}$ at the filament centre and it increases to $0.5^{\prime}$ in the more diffuse regions, where the stellar density is higher.

Afterwards, we have computed the filament extinction profile. In order to obtain the lower uncertainties, we have averaged the profile in $2^{\prime} \times 2^{\prime}$ grid, except at the filament centre (Fig. 7). The uncertainties decrease from the centre toward the filament edges, where the density of stars increases. The final angular resolution of the column density profile is $3.5^{\prime}$ at the centre (offsets $<1.75^{\prime}$ ) and $2^{\prime}$ elsewhere. The cell covering the central area of the filament has an inhomogeneous distribution of stars. Two stars are located in the centre while the rest is located towards the edge of the cell. We therefore consider the computed value of $A_{v}$ in the central $3.5^{\prime}$ diameter area as a lower limit.

\section{Analysis}

\subsection{Temperature determination}

We separate the cloud emission into two components, a bright filament and a surrounding envelope. The surrounding envelope is fitted using a second order polynomial (dashed lines in Fig. 2). The bright filament is obtained after subtraction of the envelope emission. The resulting profiles together with the IRAS beam profile at 100 micron are presented in Fig. 3. We see that the filament is resolved with a width of $8^{\prime}$ (FWHM) at $260 \mu \mathrm{m}$. The width of the filament slightly decreases with increasing wavelength (Fig. 3). Moreover, the 100 and $200 \mu \mathrm{m}$ profiles present a significant excess for angular offsets of $4^{\prime}-10^{\prime}$ ( $4 \sigma$ and $3 \sigma$ respectively).

The dust emission is fit by the following single modified black body:

$I_{\lambda}^{\mathrm{fit}}=\epsilon_{250 \mu \mathrm{m}} \times\left(\frac{\lambda}{250 \mu \mathrm{m}}\right)^{-\beta} \times B_{\lambda}\left(T_{\text {dust }}\right)$

where $\epsilon_{250 \mu \mathrm{m}}$ is the dust emissivity at $250 \mu \mathrm{m}, \beta$ the emissivity spectral index, and $T_{\text {dust }}$ the dust temperature. The parameters $\epsilon_{250 \mu \mathrm{m}}, \beta$, and $T_{\text {dust }}$ are obtained by minimising

$\chi^{2}=\sum_{i}\left(\frac{I_{i}^{\mathrm{fit}}-I_{i}^{\mathrm{obs}}}{\sigma_{i}}\right)^{2}$

All brightness values have been colour-corrected using the actual filter transmission and a grey body spectrum of the form $I_{\lambda}=\epsilon_{\lambda_{0}}\left(\lambda / \lambda_{0}\right)^{-\beta} \times B_{\lambda}\left(T_{\text {dust }}\right)$ with $\beta$ and $T_{\text {dust }}$ derived iteratively with the best fit (values in Table 3 ).

\subsubsection{Filament}

The spectrum of the brightest position of the filament is presented in Fig. 5 (Table 2). The $1 \sigma$ error is the quadratic average of the calibration error ( $5 \%$ of the signal for PRONAOS/SPM and $10 \%$ for IRAS) and the rms dispersion of the scan average, essentially due to instrumental oscillations (Sect. 3.1). The results of the fitting procedure (see above, Sect. 2.6) are given in Table 3. The best fit is obtained for $T_{\text {dust }}=12.1_{-0.1}^{+0.2} \mathrm{~K}$ and $\beta=1.9 \pm 0.2$. An emissivity index of $\beta=2$ is within the error bars. In the following, we set the value of $\beta$ to 2 , according to the value proposed by Boulanger et al. (1996) for the average submillimetre dust spectrum of the diffuse ISM.

Table 2. The spectral energy distribution of the filament at the brightest position measured by PRONAOS/SPM and IRAS.

\begin{tabular}{ccccccc}
\hline \hline$\lambda(\mu \mathrm{m})$ & 60 & 100 & 200 & 260 & 360 & 580 \\
\hline signal $(\mathrm{MJy} / \mathrm{sr})$ & $<0.15$ & 5.8 & 65.9 & 79.9 & 55.2 & 20.4 \\
$1 \sigma$ error $(\mathrm{MJy} / \mathrm{sr})$ & - & 1.0 & 4.7 & 4.4 & 3.2 & 1.3 \\
\hline
\end{tabular}

The brightness profiles (Fig. 3) indicate a temperature gradient across the filament from the centre toward the edges: from $12.0_{-0.1}^{+0.2} \mathrm{~K}$ to $14.2 \pm 0.5 \mathrm{~K}$ (Fig. $6 \mathrm{a}$ ).

\subsubsection{Envelope}

The temperature variation over the whole cloud is presented in Fig. 6b. The decrease in $T_{\text {dust }}$ toward the centre (offsets smaller than $8^{\prime}$ ) is due to the increasing contribution of the cold dust 


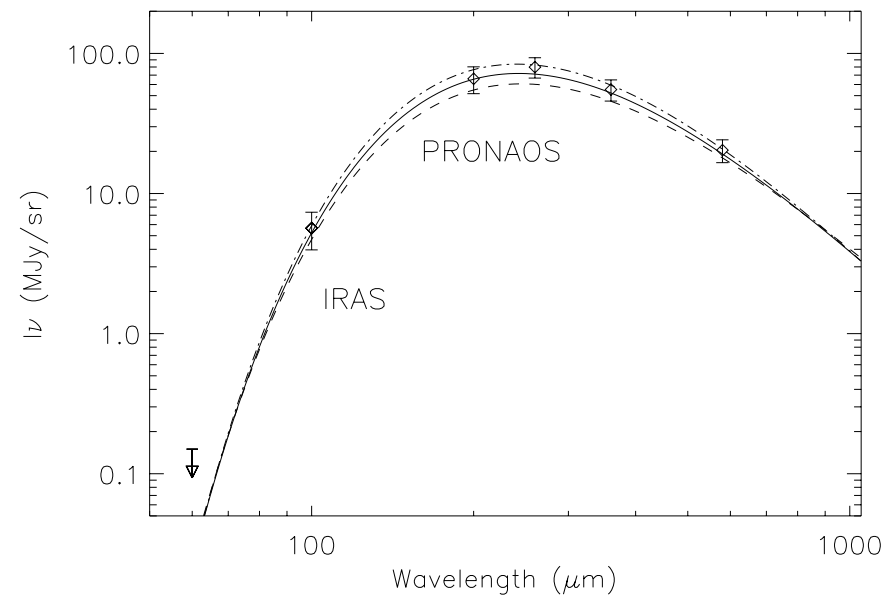

Fig. 5. Spectrum at the brightest position of the filament $\left(\alpha_{2000}=\right.$ $\left.4^{\mathrm{h}} 18^{\mathrm{m}} 50^{\mathrm{s}}, \delta_{2000}=25^{\circ} 19^{\prime} 15^{\prime \prime}\right)$. The diamonds are data points. The error bars correspond to $\pm 1 \sigma$ (Sect. 2.6.1). The solid line is our best fit $\left(T_{\text {dust }}=12.1 \mathrm{~K}, \beta=1.9\right)$, and the dashed lines are the extremal fits $\left(T_{\text {dust }}=12.3 \mathrm{~K}, \beta=1.7\right.$ and $\left.T_{\text {dust }}=12.0 \mathrm{~K}, \beta=2.1\right)$ compatible with our $1 \sigma$ error bars.

Table 3. The fitting parameters of the different cloud regions. For the envelope and the large-scale structure the spectral index $\beta$ is fixed to 2 .

\begin{tabular}{ccccc}
\hline \hline $\begin{array}{c}\text { fitted } \\
\text { parameters }\end{array}$ & filament & envelope & $\begin{array}{c}\text { large- } \\
\text { scale }\end{array}$ \\
\hline$T_{\text {dust }}(\mathrm{K})$ & $12.1_{-0.1}^{+0.2}$ & $12.0_{-0.1}^{+0.2}$ & $14.8 \pm 0.6$ & $16.8 \pm 0.7$ \\
$\beta$ & $1.9 \pm 0.2$ & 2 & 2 & 2 \\
$\epsilon_{250 \mu \mathrm{m}} \times 10^{4}$ & $34 \pm 5$ & $37 \pm 2$ & $2.9 \pm 0.3$ & $2.7 \pm 0.4$ \\
\hline
\end{tabular}

located in the filament. Therefore, we can estimate the envelope temperature only for offsets greater than $8^{\prime}$ (for negative offsets, the signal is near zero). We determine a temperature of $14.8 \pm 0.6 \mathrm{~K}$ for the envelope at offsets greater than $8^{\prime}$. For offsets smaller than $-8^{\prime}$ the signals are too weak for reliable temperature determination.

\subsubsection{Large-scale structure}

We now estimate the temperature of the large-scale structure surrounding the filament $\left( \pm 1^{\circ}\right.$ around the filament). We use DIRBE data at 140 and $240 \mu \mathrm{m}$ with an angular resulution of about one degree to derive the dust temperature outside the PRONAOS/SPM map. We subtract from the DIRBE data the foreground and background cirrus emission estimated using a linear baseline. The large scale temperature outside the filament appears constant with a value of $16.8 \pm 0.7 \mathrm{~K}$ (Table 3 ). The quoted error is the root mean square of the spatial dispersion, and the calibration error of DIRBE (5\%). This temperature is close to that of the diffuse medium $(17.5 \mathrm{~K}$; Boulanger et al. 1996), indicating that the radiation field seems to be standard outside the filament.

We conclude that we have observed significant temperature variations from $16.8 \pm 0.7 \mathrm{~K}$ outside the cloud to $12.0_{-0.1}^{+0.2} \mathrm{~K}$ inside the filament. The question is whether the attenuation of the radiation field due to extinction is the only mechanism that can
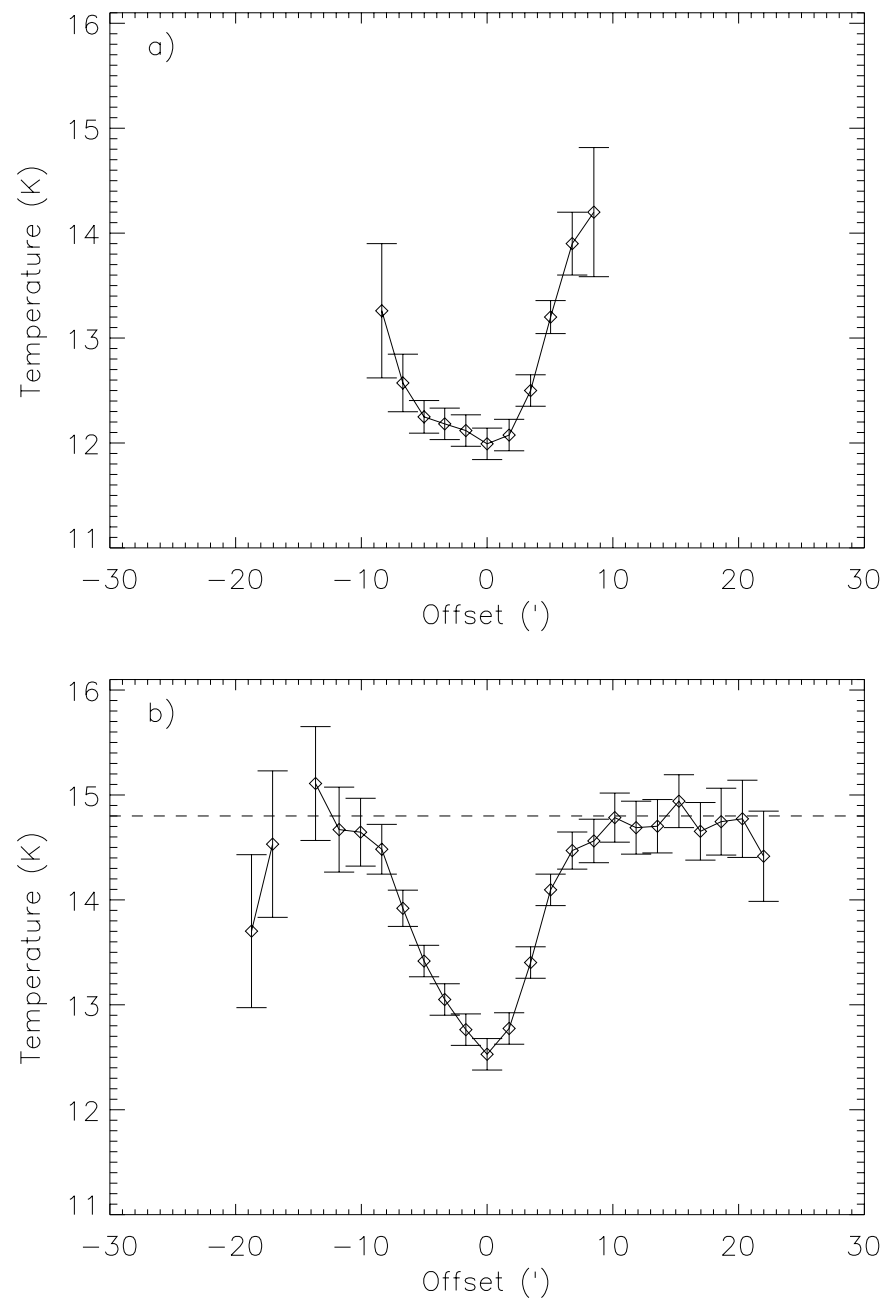

Fig. 6. Temperature profiles across the cloud (with the $1 \sigma$ error bars), fitted with a $\beta$ value of 2. Upper panel a) Filament alone (surrounding envelope subtracted.). Lower Lower panel b) Whole cloud (filament + envelope). The dashed line in Fig. $5 \mathrm{~b}$ represents $T=14.8 \mathrm{~K}$, which is the average temperature of the envelope surrounding the filament.

explain the drop in temperature. In order to answer this we have computed the filament emission using a radiative transfer code and a range of models for the dust optical properties (Sect. 5).

\subsection{Density profile}

We decompose the extinction profile into a constant large scale component, $A_{V}^{\text {large-scale }}$, and a filament component which is a function of offset from the centre, $A_{V}^{\text {filament }}(r)$.

The total extinction can be written:

$A_{V}(r)=A_{V}^{\text {large-scale }}+A_{V}^{\text {filament }}(r)$

$A_{V}^{\text {large-scale }}$ is estimated from the observed extinction at offsets $\geq 30^{\prime}$. We model the density profile inside the filament $n_{H}^{\text {filament }}(r)$ assuming a cylindrical geometry and a power law density distribution (Fig. 8):
$n_{\mathrm{H}}^{\text {filament }}(r)=n_{0}$
for $0 \leq r<r_{\mathrm{c}}$
$n_{\mathrm{H}}^{\text {filament }}(r)=n_{0} \cdot\left(\frac{r}{r_{\mathrm{c}}}\right)^{\alpha}$
for $r_{\mathrm{c}} \leq r<r_{\max }$ 
where $r_{\mathrm{c}}$ is the inner radius below which the density is constant and $r_{\max }$ the edge radius of the cloud.

$N_{H}(r)$ is converted into $A_{V}(r)$ using $N_{H} / A_{V}=1.87 \times$ $10^{21} \mathrm{~cm}^{-2} \mathrm{mag}^{-1}$, from Savage \& Mathis (1979). To reproduce the angular resolution of the observed $A_{V}$, we convolve with a Gaussian. We fit $n_{0}, r_{\mathrm{c}}$ and $r_{\max }$, to match the value of the $A_{V}$ profile measured using a $\chi^{2}$ minimisation. The value of $\alpha$ has been set to -2 , which is expected for a self-gravitating isothermal cloud. Variations in $\alpha$ do not improve the goodness of the fit, and have no significant influence on the computed emission. Since $A_{V}$ is a lower limit for $r<1.75^{\prime}$ (Sect. 3.1), we have fitted $n_{0}, r_{\mathrm{c}}$ and $r_{\max }$ for $r>1.75^{\prime}$, independently for the positive and negative offset parts of the $A_{V}$ profile. The best $A_{V}$ fit is represented in Fig. 7, the corresponding $\mathrm{n}_{\mathrm{H}}$ profile is given in Fig. 8, and the parameters in Table 4.

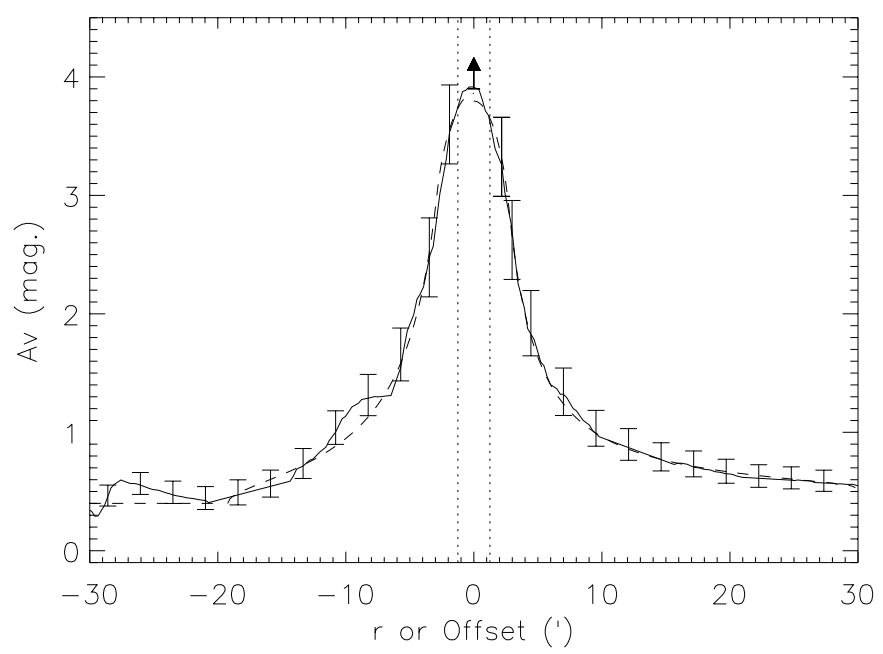

Fig. 7. $A_{V}$ profile deduced from star counts method (Sect. 3.1) and fitted with our density model (dotted line), see Sect. 3.2. The error bars presented are $1 \sigma$ error bars, and the lower limits given correspond to the central region. The vertical dotted lines delimit areas of $3.5^{\prime}$ of diameter where we have only a lower limit for the extinction.

Table 4. Density profile parameters for the fitted $A_{V}$ profile (Fig. 7).

\begin{tabular}{cccccc}
\hline \hline $\begin{array}{c}\text { angular } \\
\text { offset }\end{array}$ & $\begin{array}{c}A_{V}^{\text {large-scale }} \\
(\mathrm{mag})\end{array}$ & $\begin{array}{c}\alpha \\
-\end{array}$ & $\begin{array}{c}n_{0} \\
\left(\mathrm{H} \mathrm{cm}^{-3}\right)\end{array}$ & $\begin{array}{c}r_{\mathrm{c}} \\
\left(\mathrm{pc} /{ }^{\prime}\right)\end{array}$ & $\begin{array}{c}r_{\max } \\
\left(\mathrm{pc} /^{\prime}\right)\end{array}$ \\
\hline positive & 0.5 & -2 & 5800 & $0.095 / 2.3$ & $1.3 / 32$ \\
negative & 0.4 & -2 & 5200 & $0.11 / 2.7$ & $0.78 / 19$ \\
\hline
\end{tabular}

\section{Modelling the observed properties of the filament}

\subsection{Radiative transfer model}

We have used the 3D radiative transfer code developed by Bernard et al. (1992, 1993) and improved by Le Peintre et al. (2002). Assuming a radiation field, a cloud geometry, dust properties, and a density distribution, we can compute the grain

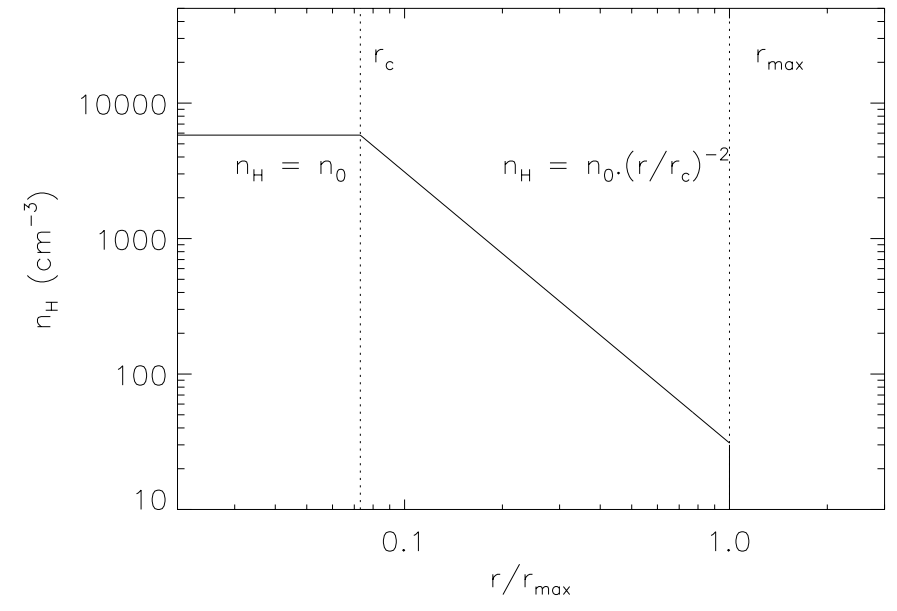

Fig. 8. The normalised density profile of the filament $\left(n_{H}^{\text {filament }}\right)$ is modelled by a power law: $n_{H}^{\text {filament }}(r)=n_{0} \cdot\left(\frac{r}{r_{\mathrm{c}}}\right)^{-2}$. The $r$ axis has been normalised to $r_{\max }$.

temperature distribution and the dust continuum emission of an interstellar cloud.

We assume that the incident radiation field is isotropic. We adopt the average interstellar radiation field (ISRF) of Mathis et al. (1983) attenuated by the visual extinction $A_{V}^{\text {large-scale }}$ determined in Sect. 3.2. The filamentary shape of the cloud is modelled assuming a cylindrical geometry. The cloud radial density distribution $n_{H}^{\text {filament }}(r)$ was determined in Sect. 3.2.

For each position inside the cloud, we compute the cloud emission in three steps. First, we calculate the attenuation of the incident radiation field. Second, we determine the temperature distribution of the dust and its emission spectrum. The dust emission is computed using the method of Désert et al. (1990). Third, we integrate the dust emission along the line of sight, taking the dust self absorption into account. The model is selfconsistent for dust extinction and emission, and includes emission from transiently-heated small grains. The results of the model are independent of the gas-to-dust ratio. The star counts provide the column density of dust and the radiative transfer model directly uses the inferred dust density distribution.

\subsection{Modelling using standard dust composition}

We assume the same dust properties throughout the cloud. We use the standard dust composition adopted by Désert et al. (1990). This is an empirical model consisting of three grain components in which the abundance and size distributions reproduce both the extinction curve and the emission spectrum measured by IRAS in the Solar neighbourhood. The three dust components are: Polycyclic Aromatic Hydrocarbons (PAHs), Very Small Grains (VSGs), and Big Grains (BGs). Both PAHs and VSGs are transiently heated by single photon absorption. While BGs are in thermal equilibrium with the radiation field.

We have integrated the emission computed by the model over the instrument filter bands, degraded the angular resolution to that of the appropriate filter band, simulated the beam modulation on the sky and deconvolved the signal as was done with the actual data (Sect. 2.4). In addition, to compare the 

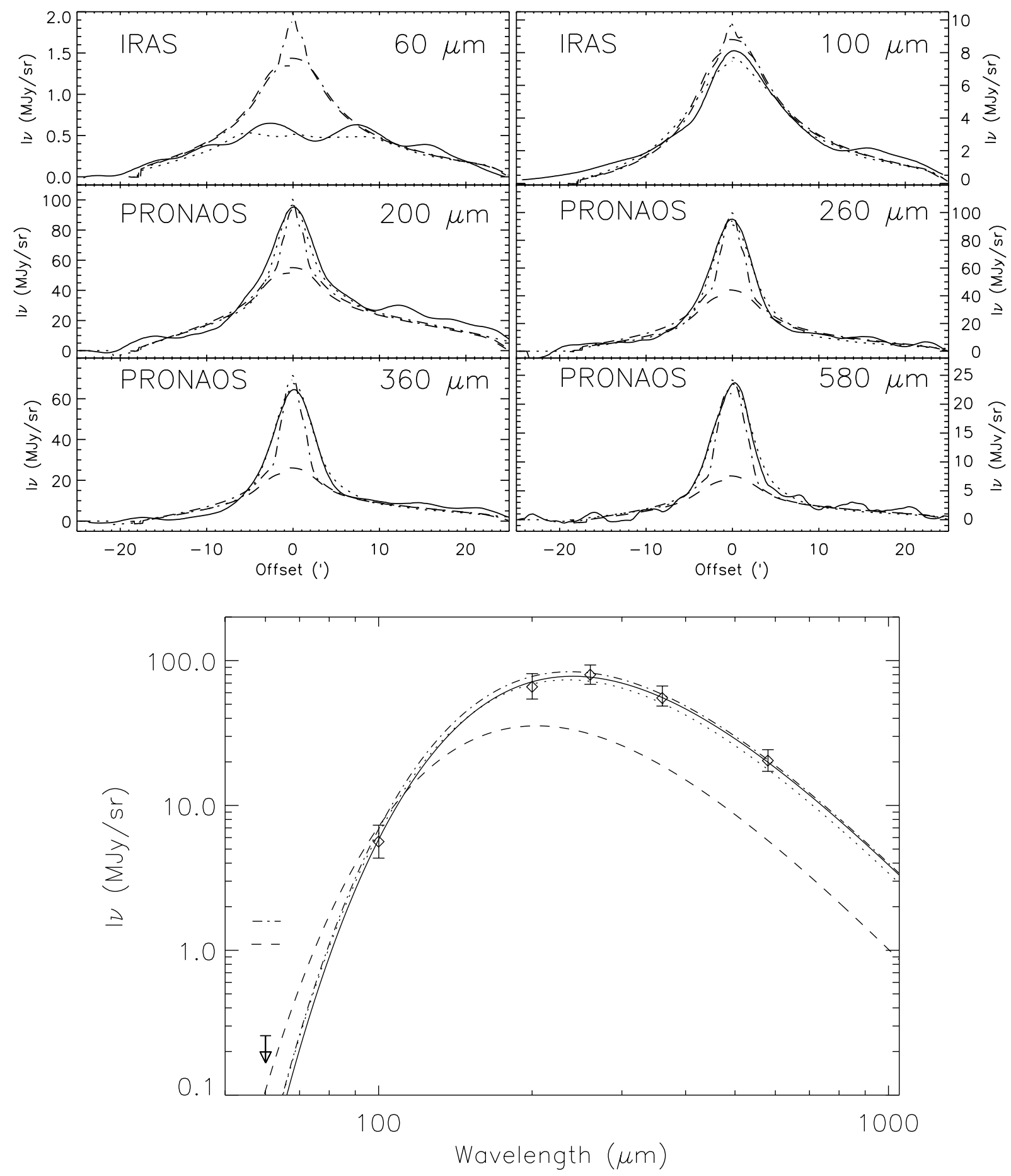

Fig. 9. We compare the data in solid lines with the models (other lines). Dashed lines are for the standard model, dash-dotted for the model using a additional central $A_{V}$ value of 35 in the central part of the filament (3.5'), and dotted lines for the model using non-standard dust properties inside the filament. Upper panel: observed and modelled brightness profiles of the filament at the angular resolution of Table 1. Lower panel: spectra of the brightest position of the filament for the different cases. Diamonds are the data points and the error bars correspond to $1 \sigma$. The dashed curve represents a $T_{\text {dust }}=14.2 \mathrm{~K}$ spectrum and the others $T_{\text {dust }} \sim 12 \mathrm{~K}$. We have also reported the filament level emission modelled at $60 \mu \mathrm{m}$ and the $1 \sigma$ upper limit measured.

model spectra with the observations, we have degraded all the computed profiles to the IRAS $100 \mu \mathrm{m}$ resolution.

The computed profiles have been plotted together with the observed profiles in Fig. 9, upper panel. Only the large scale emission is well-predicted by our model. However, at wave- lengths longer than $100 \mu \mathrm{m}$ the observed emission is too low to reproduce the observations. This is illustrated by comparing the spectra at the central position of the filament (lower part of Fig. 9), obtained by subtracting the large scale emission from the computed profiles. Toward the centre of the filament, the 
observed spectrum can be fitted with a temperature of $12 \mathrm{~K}$ (Sect. 2.6.1), while the computed spectrum gives a temperature of $14.2 \mathrm{~K}$. The model predicts a significant amount of emission at $60 \mu \mathrm{m}$ which is not observed. We conclude that the submillimetre emission profiles cannot be reproduced.

\subsection{The need for non-standard grains}

In order to better reproduce the dust temperature observed toward the centre of the filament $(12 \mathrm{~K})$, we can in principle modify the incident radiation field, the column density, or the optical properties of the dust particles. The influence of these various parameters is investigated in the following subsections.

\subsubsection{Influence of the incident radiation field}

The Taurus molecular complex is a relatively quiescent region, so we have assumed a radiation field equal to the local ISRF. Can we change the incident radiation field intensity in order to reproduce our observations? A decrease in the incident radiation field has to be compatible with the apparent dust temperature measured by DIRBE outside the cloud (offsets $\sim 1^{\circ}$ ): $16.8 \pm 0.7 \mathrm{~K}$ (Sect. 2.6.3). For the standard dust model of Désert et al. (1990) an apparent temperature of $16.8 \pm 0.7 \mathrm{~K}$ is reached for an ISRF multiplied by a factor $0.77_{-0.14}^{+0.23}$. The resulting emission profile (Fig. 9) is almost identical to the one obtained from the standard model of Sect. 4.2, and unable to reproduce our observations. The apparent dust temperature at the central position of the filament is $14.0 \mathrm{~K}$. In order to reach an apparent dust temperature of $12 \mathrm{~K}$ we have to multiply the ISRF by a factor of $\sim 0.33$. This is not compatible with the DIRBE measurements outside the cloud. The predicted envelope $\left(10^{\prime} \lesssim\right.$ offset $\left.\lesssim 30^{\prime}\right)$ temperature is too low and the amplitude of the synthetic spectrum, at the central position (offset $=0^{\circ}$ ), drops with a factor of two at all wavelengths.

Another solution is an attenuation of the incident radiation field by an additional $A_{V}$ value $\left(A_{V} \sim 1\right.$ typically). The UV part of the radiation field is more attenuated than the visible and near-IR parts, so the spectrum changes. The results of such an attenuation are (1) to decrease the temperature of the whole cloud (envelope + filament), and (2) to decrease the amplitude of the synthetic profiles at each wavelength. We conclude that we cannot reproduce the observations by changing the hardness of the radiation field.

\subsubsection{Influence of the column density profile}

The discrepancy between our model and the observations may be due to an underestimate of the column density in the centre where we measured only an upper limit for the extinction. We have increased $A_{V}$ for $r<1.75^{\prime}$ and maintained the profile found in Sect. 3.1 for larger $r$. Using the radiative transfer model, we have fitted this $A_{V}(0)$ value in order to reproduce a temperature of $12 \mathrm{~K}$ toward the centre of the filament (Fig. 9). The temperature and the amplitude of the dust spectrum are well reproduced at the peak emission, but the widths of the computed emission profiles are too narrow.
To match the data we have to increase $A_{V}$ over an area larger than $3.5^{\prime}$. Typically, a value of $A_{V}=15$ within a region of a size-scale of $7^{\prime}$ reproduces the observations. Such a profile is not compatible with the 2MASS observations, where a significant amount of stars were counted in the annulus $3.5^{\prime}<r<7^{\prime}$ (Fig. 6). The presence of these stars indicates a value of $A_{V}$ less than 15 (typically $A_{V}=4$, as seen in Fig. 7). We conclude that a change of the $A_{V}$ profile alone, within the error bars (Fig. 7), is not compatible with the observed emission profiles.

\subsubsection{Fitting the data with standard grains?}

One solution may be to change both the incident radiation field and the $A_{V}$ profile. A combination of an incident field multiplied by a factor larger than $0.63(=0.77-0.14$, the lower limit for the incident radiation field intensity, see Sect. 4.3.1) and an $A_{V}$ profile with high values in the central area of $3.5^{\prime}$ produces emission profiles which are too narrow. Therefore the only remaining option is to assume that the optical properties and the relative abundance of the grains change inside the filament.

\subsection{Modelling the filament emission with non-standard grains}

We have seen that the standard model only reproduces the observations in the envelope. For the filament, the abundance of the VSGs has to be decreased and the submillimetre emissivity must be enhanced. Two mechanisms are possible: (1) to transform the VSGs into particles emitting in the submillimetre range, and (2) to increase the BG submillimetre emissivity.

The two solutions proposed are linked, and their effects not independent. VSG coagulation onto BGs leads to a decrease of VSG abundance and at the same time changes the emission of the BGs. VSG mutual coagulation onto larger grains leads also to a decrease of VSG abundance, and may form aggregates partaking in the BG size distribution.

We introduce four new parametres in the model: $r_{\mathrm{VSG}}, f_{\mathrm{VSG}}$, $r_{\mathrm{BG}}$ and $f_{\mathrm{BG}}$. The VSG abundance is multiplied by a factor $f_{\mathrm{VSG}}(<1)$, for offsets lower than $r_{\mathrm{VSG}}$. The BG emissivity, for wavelengths greater than $20 \mu \mathrm{m}$, is multiplied by a factor $f_{\mathrm{BG}}$ $(>1)$ for offsets smaller than $r_{\mathrm{BG}}$. We use a factor $f_{\mathrm{BG}}$ independent of the wavelength in order to keep the spectral index equal to 2, as we have measured in Sect. 2.6.1. The choice of the threshold wavelength $(20 \mu \mathrm{m})$ is not critical, since BGs absorb radiation at wavelengths shorter than $10 \mu \mathrm{m}$, and emit at wavelengths greater than $50 \mu \mathrm{m}$, since they are colder than $20 \mathrm{~K}$. Variations of this wavelength threshold between 10 and $50 \mu \mathrm{m}$ changes the final BG equilibrium temperature by less than $1 \%$.

We determine the values of these four parameters by fitting the computed emission profile with the data. The error bars correspond to the different parameter values compatible with the $1 \sigma$ error bars of the emission profiles. First, we use the standard incident radiation field and the $A_{V}$ profile of Sect. 4.2.2. The $60 \mu \mathrm{m}$ deficit is reproduced with $r_{\mathrm{VSG}}=4^{\prime} \pm 1$ and $f_{\mathrm{VSG}}=0.1 \pm 0.1$, and the submillimetre enhancement is fitted with $r_{\mathrm{BG}}=4^{\prime} \pm 0.5^{\prime}$ and $f_{\mathrm{BG}}=3.4_{-0.3}^{+0.2}$. With these values, we are able to reproduce correctly the cloud emission: dust 
temperature, spectrum amplitude and profile widths at all wavelengths and at all positions (Fig. 9).

Then, we investigate the effects of different incident radiation fields and $A_{V}$ profiles. We have seen (Sect. 4.3.1) that the incident radiation field is compatible with the local ISRF multiplied by a factor down to 0.63 . The use of this lower incident radiation field slightly modifies the $f_{\mathrm{BG}}$ determination. In this case we find $f_{\mathrm{BG}}=3.0 \pm 0.2$. A change in the $A_{V}$ profile within the error bars (Sect. 3.1 and Fig. 7) also increases the uncertainty on $f_{\mathrm{BG}}$ by \pm 0.1 . The determination of $r_{\mathrm{VSG}}$ and $f_{\mathrm{VSG}}$ are not significantly modified by these changes.

Finally, the values and the error bars of the four parameters are reported in Table 5.

Table 5. The four new parameters of our model (see the text).

\begin{tabular}{cccc}
\hline $\begin{array}{c}\text { particle } \\
\text { component }\end{array}$ & & $\begin{array}{c}\text { modified } \\
\text { property }\end{array}$ \\
\hline $\mathrm{VSGs}$ & $r_{\mathrm{VSG}}=4^{\prime} \pm 1^{\prime}$ & $f_{\mathrm{VSG}}=0.1 \pm 0.1$ & abundance \\
$\mathrm{BGs}$ & $r_{\mathrm{BG}}=4^{\prime} \pm 0.5^{\prime}$ & $f_{\mathrm{BG}}=3.4_{-0.7}^{+0.3}$ & submm emissivity \\
\hline
\end{tabular}

This simple cloud model in two dust phases separated by an abrupt transition reproduces our data very well (Fig. 9). The size of the region where the dust properties are modified is the same for the BGs $\left(4^{\prime} \pm 0.5^{\prime}\right)$ and the VSGs $\left(4^{\prime} \pm 1^{\prime}\right)$ within the error bars. Therefore BG properties and VSG abundance seem to be physically connected and it is likely that a common physical process affects these two components. With our data, we cannot resolve the dust property variations inside each phase. Thus, the interface between the two phases is smaller than the beam size of our observations $\left(3.5^{\prime}\right.$ or $\left.0.14 \mathrm{pc}\right)$. Such an abrupt change in the dust properties is the signature of an efficient and fast process which appears for a threshold of $A_{V}=2.1 \pm 0.5$ (measured on the line of sight or $A_{V}^{\perp}=0.9 \pm 0.2$ when computed along the filament radial direction) and $n_{H}=3 \pm 2 \times 10^{3} \mathrm{~cm}^{-3}$.

\section{Interpretation}

We have shown that, inside the densest part of the filament, (1) the transiently heated small particles are not present, (2) the ratio of the grain submillimetre emissivity to the dust extinction in band $J$ is significantly enhanced, and (3) these two phenomena coincide spatially. We want to emphasis that our results are normalised to the value of the extinction observed in the $J$-band. In particular, an overestimate of the dust extinction in the $J$ band produces an underestimate of the dust column density, and thus an apparent submillimetre emissivity increase. In our study, we have not shown an increase in the dust submillimetre emissivity, but an increase of the ratio between the dust submillimetre emissivity over the dust extinction in $J$ band.

Several processes may affect the optical properties of dust in dense clouds: ice or molecular mantle formation on grains, coagulation of grains (e.g. Draine 1985). Preibisch et al. (1993) have shown that ice and carbon mantles on BGs do not increase significantly the ratio of submillimetre emissivity over extinction in $J$ band. Therefore, grain mantle formation cannot be the main explanation for our observations.

\subsection{Grain-grain coagulation}

Dust coagulation is efficient at producing large aggregates (Weidenschilling \& Ruzmaikina 1994; Ossenkopf 1993). These aggregates have irregular and fluffy shapes, which can be modelled by fractal grains (Meakin \& Donn 1988; Wurm $\&$ Blum 2000). The optical properties of fluffy aggregates have been computed by several authors (Bazell \& Dwek 1990; Ossenkopf \& Henning 1994; Stognienko et al. 1995). The submillimetre emissivity increases with fluffiness. At $200 \mu \mathrm{m}$ this increase is typically of a factor $1.5-3.5$ for silicate and 3-20 for carbon aggregates (e.g. Stognienko et al. 1995). The UV, visible and near-IR absorptivity are not significantly modified if the fluffiness is increased (Bazell \& Dwek 1990). As a result, the equilibrium temperature of fluffy aggregates is lower than that of compact particles. Fogel \& Leung (1998) have found a temperature decrease of typically 10-20\%, for the grains of Bazell \& Dwek (1990), heated by the local ISRF.

Coagulation simulations show that smallest grains (VSGs) coagulate faster than larger ones (Ossenkopf 1993). This can explain the strong decrease of the VSG abundance observed. We conclude that grain-grain coagulation into fluffy aggregates can produce both the strong variations of relative abundance and optical properties of dust observed in the Taurus filament.

\subsection{Cloud lifetime and coagulation timescales}

The cloud lifetime is at least equal to the free-fall timescale of the cloud (e.g. Walmsley 1991):

$\tau_{\text {free-fall }}=\left(\frac{3 \pi}{32 G n_{H}}\right)^{-\frac{1}{2}}$.

The local density for $r>1.75^{\prime}$ is around $4 \times 10^{3} \mathrm{~cm}^{-3}$ (Sect. 3.2). For such densities the cloud lifetime is above $10^{6}$ years.

The coagulation timescale between two grains (grain type 1 on grain type 2) is estimated by the relation (e.g. Draine 1985):

$\tau_{\text {coa }}=\left[\sigma_{1 / 2} \times n_{1} \times v_{1 / 2}\right]^{-1}$

where $\sigma_{1 / 2}$ is the coagulation cross-section, $n_{1}$ the density of grains type 1 , and $v_{1 / 2}$ the relative velocity between the two grains. For an estimate, we consider the coagulation cross section equal to the geometric cross section: $\sigma_{1 / 2}=\pi \times\left(a_{1}+a_{2}\right)^{2}$, where $a_{1}$ and $a_{2}$ are the radii of grains 1 and 2 .

We compute the average grain radius $\langle a\rangle$ and corresponding density $n_{\langle a\rangle}$ for the VSG and BG components in the standard dust model (Table 6). The resulting values of $\sigma_{1 / 2}$ and $n_{1}$ are given in Table 7.

There is a critical velocity above which collisions do not lead to coagulation but destruction (Chokshi et al. 1993). This critical velocity depends on the size and the composition of porous grains (Dominik \& Tielens 1997). Icy grains stick better than other particles, because their mantles can absorb a significant fraction of the collisional energy. Their critical velocity is 
Table 6. Average grain radii $\langle a\rangle$ and corresponding densities $n_{\langle a\rangle}$ computed from the standard dust model (see Table 2 of the Désert et al. 1990 paper).

\begin{tabular}{ccc}
\hline \hline dust component & $\langle a\rangle$ & $n_{\langle a\rangle} / n_{H}$ \\
\hline VSG & $3 \mathrm{~nm}$ & $5 \times 10^{-9}$ \\
BG & $30 \mathrm{~nm}$ & $3 \times 10^{-11}$ \\
\hline
\end{tabular}

higher than for naked grain aggregates, causing the coagulation timescale to become smaller than that of naked grain aggregates. By studying ice mantle formation in the Taurus molecular complex, Texeira \& Emerson (1999) have found a linear relationship between the width of the ice mantles and $A_{V}$, and derived a threshold column density of $A_{v}=2-3$ for the detection of ice mantles. Towards the filament, the submillimeter excess is detectable in regions where $A_{V} \gtrsim 2$ suggesting that grains are coated with ice mantles.

We have calculated the critical velocity of ice grains according to Dominik \& Tielens (1997), and found:

$v_{\text {critical }}=670 \times\left[\left(\frac{1}{a_{1}}+\frac{1}{a_{2}}\right) \times 10^{-5} \mathrm{~cm}\right]^{\frac{5}{6}} \mathrm{~cm} \mathrm{~s}^{-1}$.

Numerical results are given in Table 7. Brownian motions lead to very low relative velocities between grains of about $1 \mathrm{~m} / \mathrm{s}$ (Draine 1985). These relative velocities are not efficient at producing grain coagulation. However, the presence of turbulent motions (Falgarone \& Phillips 1990) can enhance the relative grain velocity. Volk et al. (1980) and Draine (1985) have analysed relative grain velocities in turbulent clouds with a Kolmogorov spectrum of turbulent eddies. Typically, relative grain velocities of about $1 \mathrm{~km} \mathrm{~s}^{-1}$ are expected for 3-30 nm grains at a density of $4 \times 10^{3} \mathrm{~cm}^{-3}$ in molecular clouds (Tielens 1989). This value is roughly equal to the critical velocities of Table 7 . Therefore, we assume that the relative grain-grain velocity $\left(v_{1 / 2}\right)$ inside the filament is equal to the critical velocity between grains $\left(v_{\text {critical }}\right)$. The coagulation timescales for the possible interactions between VGSs and BGs are also presented in Table 7 . The values of $\sigma_{1 / 2}, n_{1}$ and $v_{\text {critical }}$ are reported in order to underline the relative importance of these factors. In Table 7, "VSGs on BGs" means coagulation of VSGs onto BGs. The coagulation timescale of a VSG onto one BG is different from that of a BG onto a VSG. In the former case the $\mathrm{BG}$ is the choosen target grain, and the difference in timescales arises from the abundance of the incident grains.

The coagulation timescales computed above are simply the time that one type of grain meets another. However, the shortest timescale will dominate the coagulation process. The timescale for VSGs onto BGs is the smallest and results from a combination of (1) high velocities, (2) the large number of small grains, and (3) the large cross-section of the large grains. The total timescale to form one coagulated grain, to first order, has to be multiplied by the number of VSGs that have stuck to a BG. How many VSG particles are requiered to modify the optical properties of a BG particule? Bazell \& Dweck (1990) have computed a submillimetre excess of 5 for fluffy aggregates of
Table 7. Dust parameters and their resulting coagulation timescales.

\begin{tabular}{ccccc}
\hline \hline coagulation & $\begin{array}{c}\sigma_{1 / 2} \\
\left(\mathrm{~cm}^{2}\right)\end{array}$ & $\begin{array}{c}n_{1} \\
\left(\mathrm{~cm}^{-3}\right)\end{array}$ & $\begin{array}{c}v_{\text {critical }} \\
\left(\mathrm{km} \mathrm{s}^{-1}\right)\end{array}$ & $\begin{array}{c}\tau_{\text {coa }} \\
(\mathrm{yr})\end{array}$ \\
\hline VSGs on VSGs & $1.1 \times 10^{-12}$ & $2 \times 10^{-5}$ & 2.2 & $7 \times 10^{4}$ \\
VSGs on BGs & $3.4 \times 10^{-11}$ & $2 \times 10^{-5}$ & 1.3 & $4 \times 10^{3}$ \\
BGs on VSGs & $3.4 \times 10^{-11}$ & $1.2 \times 10^{-7}$ & 1.3 & $6 \times 10^{5}$ \\
BGs on BGs & $1.1 \times 10^{-10}$ & $1.2 \times 10^{-7}$ & 0.3 & $8 \times 10^{5}$ \\
\hline
\end{tabular}

136 grains of amorphous carbon of $10 \mathrm{~nm}$. We say that it typically requires less than 100-200 VSGs to significantly alter the optical properties of BG particles. The coagulation timescale to form such aggregates is smaller than the cloud lifetime. We conclude from this simplified calculation that grain-grain coagulation is a likely process and that VSGs coagulation onto BGs could be the main mechanism to naturally explain the observations.

\subsection{Other observations}

Laureijs et al. (1991) measured, using IRAS data, strong emission deficits at $60 \mu \mathrm{m}$ toward the translucent cloud L1780 ( $A_{B} \sim 4$, note that $A_{V} / A_{B}=0.75$ from Cardelli et al. 1989) and the dark clouds L183 and L134 $\left(A_{B} \sim 10\right)$. In these clouds, a significant deficit of the $60 \mu \mathrm{m}$ emission flux appears for $A_{B} \gtrsim 2$ and $n_{H} \gtrsim 10^{3} \mathrm{~cm}^{-3}$, which is in the range of our threshold values (Sect. 4.4).

Using PRONAOS/SPM data, Bernard et al. (1999) observed a low equilibrium temperature toward a high latitude cirrus cloud (MCLD 123.5+24.9), presenting a strong deficit of its $60 \mu \mathrm{m}$ emission. The apparent equilibrium temperature with the radiation field is $T=13.4 \mathrm{~K}$ (assuming $\beta=2$ ), while a radiative transfer model, using standard grains properties, predicts $15.6 \mathrm{~K}$ (with $\beta=2$ ). Bernard et al. (1999) proposed a change in the dust properties and the VSG coagulation to explain this unusual cold temperature in a cirrus. We find that an enhancement of the submillimetre emissivity by 2.8 in this cirrus produces an apparent temperature of $13.4 \mathrm{~K}$.

On larger scales, Cambrésy et al. (2001) recently decomposed the far-infrared flux of the Polaris Flare into a cold component and a warm component, defined by the $I_{60 \mu \mathrm{m}} / I_{100 \mu \mathrm{m}}$ flux ratio following the method of Lagache et al. (1998). The comparison of the far-infrared cold component with extinction maps derived from star counts indicates an enhancement of the far-infrared emissivity by a factor of $\sim 3$. In the Polaris Flare they measured the same observational effects that we have seen in the Taurus filament, but for a diffuse cloud $\left(A_{V}<1\right)$. However, we want to emphasise that the Polaris Flare is a very particular region. It is a molecular region (Heithausen \& Thaddeus 1990) and probably material reprocessed by a supernova shock (Meyerdierks et al. 1991).

Lagache et al. (1998) analysed the far-IR and submm emission of the Taurus molecular complex on large scales with DIRBE data (angular resolution $\sim 1^{\circ}$ ). They have measured temperatures of $16-17 \mathrm{~K}$ in the diffuse parts of the Taurus 
complex. The molecular filaments in this complex shows an average temperature of $13.4 \mathrm{~K}$. We suggest that the average temperature of $13.4 \mathrm{~K}$ in molecular regions is due to a mixture of $12 \mathrm{~K}$ in the dense regions with the diffuse medium at $16.5 \mathrm{~K}$. Based on this assumption, we can reproduce the large scale DIRBE emission at $240 \mu \mathrm{m}$ in this region. We suggest that the submillimetre emissivity could be enhanced in the central parts of all molecular regions by a factor of 3 (where typically $n_{H}>10^{3} \mathrm{~cm}^{-3}$ ) in order to explain the observed temperatures.

These observations indicate that in our galaxy the $60 \mu \mathrm{m}$ deficit seems to be associated with grain submillimetre emissivity enhancements by factors of 3 , pointing towards dust evolution as a general process in the denser parts of molecular clouds.

\section{Conclusions}

From PRONAOS/SPM data, IRAS data and extinction measurements, coupled with a radiative transfer model, we have shown that the dust properties change in a molecular filament $\left(A_{V} \sim 4\right)$. We have shown the presence of non-standard dust properties inside the densest parts of one filament in Taurus. We have not been able to resolve the transition between standard and non-standard grains properties with an angular resolution of $3.5^{\prime}$ (corresponding to $0.14 \mathrm{pc}$ ). This transition appears for threshold values of $A_{V}=2.1 \pm 0.5$ and $n_{H}=3 \pm 1 \times 10^{3} \mathrm{~cm}^{-3}$. The non-standard dust composition is characterised by:

- a 80-100\% deficit of the transiently heated grains emitting at $60 \mu \mathrm{m}$ (VSGs);

- an increase of the ratio of grain submillimetre emissivity over grain extinction in the $J$ band by a factor $3.4_{-0.7}^{+0.3}$.

These changes in dust properties also explain the cold equilibrium temperature of $12 \mathrm{~K}$ observed towards the filament centre.

We explain our observations by introducing grain-grain coagulation into fluffy aggregrates as an important process inside the cloud. Coagulation can cause an abundance deficit of VSGs and a submillimetre excess compatible with our observations.

Grain-grain coagulation could be a part of a general evolution process which modifies the properties of dust in the dense ISM. The main consequences are:

- an increase in the dust opacity per unit of mass by a factor around 3 in the dense ISM (Ossenkopf \& Henning 1994). Cloud masses derived from submillimetre measurements could be overestimated by the same factor using standard grain properties;

- modifications of the statistical properties of the submillimetre fluctuations in molecular regions (Abergel et al. 1996);

- the emission of dust particles cannot easilly be extrapolated from measurements at wavelengths shorter than $100 \mu \mathrm{m}$ or from submillimetre measurements at large angular scale. As a consequence, the removal of the galactic emission ("foregrounds") for cosmological surveys in the submillimetre is not trivial, even out of the galactic plane.

All these effects will be analysed over a large fraction of the sky by future balloon missions such as ELISA
(Ristorcelli et al. 2001) and future satellite missions such as Herschel Observatory and Planck.

In a forthcoming paper (Stepnik et al. 2003) we will analyse the optical properties of coated grains and coagulated aggregates in order to better understand the dust grain physical evolution with density.

Acknowledgements. We are indebted to the French space agency CNES who supported the PRONAOS/SPM project. We are very grateful to the CNRS and CNES PRONAOS technical teams.

We thank the NASA-NSBF balloon launching facilities group in FortSumner (New-Mexico).

We are grateful to Dr. Désert for having used his dust model code.

We thank Dr. Laureijs for very helpful comments and improvements to the manuscript.

This publication makes use of data products from the Two Micron All Sky Survey, which is a joint project of the University of Massachusetts and the Infrared Processing and Analysis Center/California Institute of Technology, funded by the National Aeronautics and Space Administration and the National Science Foundation.

\section{References}

Abergel, A., Boulanger, F., Mizuno, A., et al. 1994, ApJ, 423, 59

Abergel, A., Boulanger, F., Delouis, J. M., et al. 1996, A\&A, 309, 245

Bazell, D., \& Dwek, E. 1990, ApJ, 360, 142

Bernard, J.-P., Boulanger, F., Désert, F. X., et al. 1992, A\&A, 263, 258

Bernard, J.-P., Boulanger, F., \& Pujet, J.-L. 1993, A\&A, 277, 609

Bernard, J.-P., Abergel, A., Ristorcelli, I., et al. 1999, A\&A, 347, 640

Boulanger, F., Falgarone, E., Pujet, J.-L., et al. 1990, ApJ, 364, 136

Boulanger, F., Abergel, A., Bernard, J.-P., et al. 1996, A\&A, 312, 256

Buisson, F., \& Durand, M. 1990, in ESA: From Ground-Based to Space-Borne Sub-mm Astronomy, 323

Cambrésy, L. 1999, A\&A, 345, 965

Cambrésy, L., Boulanger, F., Lagache, G., et al. 2001, A\&A, 375, 999

Cardelli, J., Clayton, G. C., \& Mathis, J. S. 1989, ApJ, 345, 245

Chokshi, A., Tielens, A. G. G. M., \& Hollenbach, D. 1993, ApJ, 407, 806

Cutri, R. M., Skrutskie, M. F., Van Dyk, S., et al. 2000, Explanatory Supplement to 2MASS Second Incremental Data Release

Désert, F.-X., Boulanger, F., \& Pujet, J.-L. 1990, A\&A, 237, 215

Draine, B. T., \& Lee, H. M. 1984, ApJ, 285, 89

Draine, B. T. 1985, in From protostars \& Planets II, ed. D. C. Black, 621

Dominik, C., \& Tielens, A. G. G. M. 1997, ApJ, 480, 647

Elias, J. H. 1978, ApJ, 224, 857

Emerson, D. T., Klein, U., \& Haslam, C. G. T. 1979, A\&A, 76, 92

Falgarone, E., \& Phillips, T. G. 1990, ApJ, 359, 344

Fogel, M. E., \& Leung, C. M. 1998, ApJ, 501, 175

Hauser, M. G., Kelsall, T., Leisawitz, D., \& Weiland, J. 1997, DIRBE Explanatory Supplement, COBE Ref. Pub. No 97-A

Heithausen, A., \& Thaddeus, P. 1990, ApJ, 353, L49

Kenyon, S. J., Dobrzycka, D., \& Hartmann, L. 1994, AJ, 108, 1872

Lagache, G., Abergel, A., Boulanger, F., et al. 1998, A\&A, 333, 709

Lamarre, J.-M., Pajot, F., Torre, J.-P., et al. 1994, IR Phys. Tecno., 35, 277

Laureijs, R. J., Clark, F. O., \& Prusti, T. 1991, ApJ, 371, 602

Le Peintre, F., et al. 2002, in preparation 
Mathis, J. S., Mezger, P. G., \& Panagia, N. 1983, A\&A, 267, 119

Mathis, J. S. 1990, ARA\&A, 28, 37

Meakin, P., \& Donn, B. 1988, ApJ, 329, L39

Meyerdierks, H., Heithaussen, A., \& Reif, K. 1991, A\&A, 245, 247

Ossenkopf, V. 1993, A\&A, 280, 617

Ossenkopf, V., \& Henning, T. 1994, A\&A, 291, 943

Pajot, F., Stepnik, B., Lamarre, J.-M., et al. 2002, in preparation

Preibisch, T., Ossenkopf, V., Yorke, H. W., et al. 1993, A\&A, 279, 577

Ristorcelli, I., Serra, G., Lamarre, J.-M., et al. 1998, ApJ, 496, 267

Ristorcelli, I., Bernard, J.-P., Stepnik, B., et al. 2001, Proc. of The Promise of FIRST Symp., ESA SP-460

Savage, A., \& Mathis, J. S. 1979, ARA\&A, 17, 73

Serra, G., Sirmain, C., Lamarre, J. M., et al. 1997, Proc. of International Conference of Space Optics (ICSO)

Schlegel, D. J., Finkbeiner, D. P., \& Davis, M. 1998, ApJ, 500, 525
Sellgren, K., Allamandola, L. J., Bregman, J. D., et al. 1985, ApJ, 299, 416

Stognienko, R., Henning, T., \& Ossenkopf, V. 1995, A\&A, 296, 797

Teixeira, T. C., \& Emerson, J. P. 1999, A\&A, 351, 292

Tielens, A. G. G. M. 1989, Proc. IAU Symp. 135, ed. L. J. Allamandola, \& A. G. G. M. Tielens (Kluwer Academic Publishers, Dordrecht), 239

Torre, J.-P., \& Chanin, G. 1985, Rev. Scient. Inst., 26, 328

Volk, H. J., Jones, F. C., Morfill, G. E., et al. 1980, A\&A, 85, 316

Walmsley, C. M. 1991, in Fragmentation of Molecular Clouds and Star Formation, IAU Symp., 147, 161

Weidenschilling, S. J., \& Ruzmaikina, T. V. 1994, ApJ, 430, 713

Wheelock, S., Gautier, T. N., Chilleni, J., et al. 1998, IRAS Sky Survey Atlas: Extraplanatory Supplement

Wurm, G., \& Blum, J. 2000, ApJ, 529, L57 